\title{
Técnicas de mineração de dados aplicadas à classificação do estádio sucessional da vegetação em áreas de floresta ombrófila mista
}

\section{Applying data mining techniques to the classification of successional forest stages mixed shade tolerant forest environments}

\author{
Camile Sothe1, Marcos Benedito Schimalski², Veraldo Liesenberg², \\ Cláudia Maria de Almeida ${ }^{3}$, Camila Furlan de Souza ${ }^{4}$ e João Boing de Souza ${ }^{5}$
}

\begin{abstract}
Resumo
Este trabalho teve como objetivo avaliar diferentes aplicativos open source relacionados à análise baseada em objeto e mineração de dados, para a classificação de estádios sucessionais de florestas secundárias da Floresta Ombrófila Mista (FOM) em duas áreas-teste no planalto sul catarinense. Foram utilizadas ortoimagens do Sistema Aerotransportado de Aquisição e Pós-processamento de Imagens (SAAPI) de alta resolução espacial $(0,39 \mathrm{~m})$. Os dados consistem de três bandas no espectro visível $(0,38-0,70 \mu \mathrm{m})$, três bandas no espectro do infravermelho próximo $(0,76-0,78 \mu \mathrm{m})$ e o Modelo Digital de Superfície (MDS). As metodologias foram desenvolvidas utilizando mineração de dados com algoritmos de árvore de decisão e seleção de atributos nos aplicativos InterIMAGE, WEKA e QGIS. Os resultados se mostraram satisfatórios para classificar estádios sucessionais da FOM, assim como outras classes de uso e cobertura da terra. As classificações apresentaram índices Kappa variando entre 0,53 e 0,59 e Kappa condicional variando entre 0,29 e 0,83 para os estádios sucessionais da vegetação. Estes resultados demonstram o potencial dessas abordagens na extração de informações de imagens de alta resolução espacial, bem como a possibilidade de fornecer subsídios para a implementação de políticas públicas e no monitoramento dos recursos florestais.
\end{abstract}

Palavras-chave: Sucessão florestal, Árvores de decisão, Imagens aerotransportadas.

\begin{abstract}
This study aimed to evaluate open source softwares in order to classify secondary successional forest stages in Shade Tolerant Mixed Forest (FOM) environments in Southern Brazil. Two test sites were selected in the mountainous region of Santa Catarina State. We used scenes from the airborne system for acquisition and post-processing of images (SAAPI) with a spatial resolution of $0.39 \mathrm{~m}$. The dataset consists of orthorectified images containing three spectral bands in the visible range (i.e. $0.38-0.70 \mu \mathrm{m}$ ), three spectral bands in the near infrared (i.e. $0.76-0.78 \mu \mathrm{m}$ ) and a digital surface model. The methodologies were developed using feature selection and decision tree algorithms in the following open source softwares: InterIMAGE, WEKA and QGIS. The results were satisfactory to classify successional stages of FOM as well as other classes of land use and land cover. The obtained Kappa indices ranged from 0.53 to 0.59 and the conditional Kappa varied from 0.29 to 0.83 for the successional forest stages. These results demonstrate the potential of these approaches for the extraction of information in high spatial resolution imagery as well as the possibility of providing subsidies for the implementation of public policies and monitoring of forest resources.
\end{abstract}

Keywords: Secondary forests; Decision tree; Aerophotogrammetric survey.

\footnotetext{
${ }^{1}$ Mestre em Engenharia Florestal. UDESC - Universidade do Estado de Santa Catarina / Centro Agroveterinário. Av. Luiz de Camões, 2090 - Conta Dinheiro - 88520000 - Lages, SC, Brasil. E-mail: camilesothe@yahoo.com.br.

2Professor adjunto no departamento de Engenharia Florestal. UDESC - Universidade do Estado de Santa Catarina / Centro Agroveterinário. Av. Luiz de Camões, 2090 - Conta Dinheiro - 88520000 - Lages, SC, Brasil. E-mail: a2mbs@cav.udesc.br; veraldo@gmail.com.

${ }_{3}^{3}$ Pesquisadora na Divisão de Sensoriamento Remoto-DSR. INPE- Instituto Nacional de Pesquisas Espaciais / Coordenação Geral de Observação da Terra. Av. dos Astronautas, 1758 - Jardim da Granja - 12227010 - São José dos Campos, SP, Brasil. E-mail: almeida@dsr.inpe.br

${ }^{4}$ Mestrando em andamento em Engenharia Florestal. UDESC - Universidade do Estado de Santa Catarina / Centro Agroveterinário. Av. Luiz de Camões, 2090 - Conta Dinheiro - 88520000 - Lages, SC, Brasil. E-mail: milahfurlan@gmail.com.

${ }^{5} \mathrm{Graduando}$ em andamento em Engenharia Florestal. UDESC - Universidade do Estado de Santa Catarina / Centro Agroveterinário. Av. Luiz de Camões, 2090 - Conta Dinheiro - 88520000 - Lages, SC, Brasil. E-mail: falcmalvinus@gmail.com.
} 


\section{INTRODUÇÃO}

O mapeamento dos remanescentes florestais da Mata Atlântica e seus estádios de sucessão consiste em uma etapa fundamental para implantação de diversos estudos, ações de fiscalização e manejo ambiental (RIBEIRO et al., 2009). A proposição de metodologias que contemplem essa temática permite avaliar quantitativamente e qualitativamente os fragmentos remanescentes bem como a sua distribuição espacial. Essas informações podem subsidiar estudos complementares, a exemplo do estabelecimento de áreas prioritárias para conservação, entre outros (AMARAL et al., 2009). Uma vez que os estádios sucessionais são mencionados nos textos das leis de proteção ambiental, a exemplo da Lei da Mata Atlântica (Lei 11.428/2006) (BRASIL, 2006), torna-se, portanto, necessário avaliar o papel de dados remotamente situados para atendimento da legislação vigente.

No mapeamento de estádios de sucessão florestal, os espectros de reflectância são muito similares, tornando o processo de classificação uma tarefa desafiadora (VIEIRA et al., 2003). Portanto, a pesquisa de técnicas e metodologias de classificação que contemplem atributos relacionados à forma do alvo, à textura ou relações entre alvos vizinhos no mapeamento das fases de sucessão florestal ainda é necessária.

Neste contexto, o advento de novos sistemas sensores e a melhoria das resoluções radiométrica, espectral, temporal e espacial impulsionaram o surgimento de novas metodologias para extração de informações, superando as limitações das metodologias tradicionais existentes (pixel-a-pixel e por regiões). A segmentação das imagens ganhou destaque quando foi incorporada à análise baseada em objeto (Object-based Image Analysis- OBIA) (BLASCHKE; STROBL, 2001). Nela, os pixels não só são agrupados em segmentos, mas também são reconhecidos como objetos. Os objetos, diferentemente das regiões ou segmentos, são dotados de significado e identidade, sendo distinguíveis, portanto, pela sua própria existência e não pelas propriedades que possuem. Além das propriedades espectrais, espaciais e texturais, os objetos apresentam relações contextuais e semânticas, que podem ser utilizadas para a análise de imagens e aproximam-se dos processos cognitivos humanos de interpretação de imagens (CAMARGO et al., 2009).

A rede semântica, que expressa um modelo de conhecimento e se materializa graficamente em uma estrutura hierárquica de classes, pode ser gerada de forma heurística pelo usuário, testando iterativa e interativamente os descritores, as funções e os seus limiares para a discriminação adequada dos alvos. Pode também ser gerada de forma automática, através da aplicação de técnicas de mineração de dados (FRANCISCO; ALMEIDA, 2012). Estas consistem na extração de conhecimento de uma base com um grande volume de dados por meio de métodos inteligentes. O modelo derivado da mineração pode ser representado de várias formas, entre elas, as árvores de decisão, constituídas por um fluxograma com estrutura de árvore e facilmente convertidas em regras de classificação (HAN; KAMBER, 2006).

Diante disso, este estudo visou à classificação do uso e cobertura da terra em ortoimagens produzidas pelo Aerolevantamento Fotogramétrico de Santa Catarina (SC) e, através de técnicas de mineração de dados, identificar os principais atributos que diferenciam os estádios sucessionais da vegetação em áreas de Floresta Ombrófila Mista (FOM). Para isto, testaram-se algoritmos de árvore de decisão e seleção de atributos, nos sistemas open source Interpreting Images Freely (InterIMAGE), Waikato Environment Knownledge Analysis (WEKA) e Quantum Geographic Information System (QGIS). Com isso, pretendeu-se analisar a aplicabilidade das informações extraídas para as áreas de fiscalização, gestão, manejo e recuperação ambiental.

\section{MATERIAL E MÉTODOS}

O estudo foi realizado em duas áreas pertencentes à região fitoecológica de FOM (KLEIN, 1978), situada na mesorregião Serrana e microrregião Campos de Lages do estado de SC (IBGE, 2015). A área-teste A está localizada no município de Anita Garibaldi, nas coordenadas geodésicas médias $27^{\circ} 41^{\prime} 21^{\prime \prime}$ sul e $51^{\circ} 07^{\prime} 48^{\prime \prime}$ oeste. A área-teste B é localizada em Urubici, no interior do Parque Nacional de São Joaquim (i.e., $28^{\circ} 00^{\prime} 54^{\prime \prime}$ sul e $49^{\circ} 35^{\prime} 30^{\prime \prime}$ oeste), e, por esse motivo, possui menor influência antrópica que a área-teste A. Segundo a classificação de Köppen, o clima das duas áreas-teste é do tipo "Cfb", clima temperado, com verão ameno e chuvas uniformemente distribuídas. 
O levantamento aerofotogramétrico no estado de SC foi executado com recursos da Secretaria de Estado do Desenvolvimento Econômico Sustentável (SDS) entre 2010 e 2011. Esse levantamento foi realizado com um Sistema Aerotransportado de Aquisição e Pós-processamento de Imagens Digitais (SAAPI), com sensor CCD (Charge Coupled Device, "dispositivo de carga acoplado"), resolução geométrica de $39 \mathrm{~cm}$, e filtro UV-Sky, que filtra a luz ultravioleta e compensa efeitos atmosféricos (PIAZZA et al., 2016).

Os produtos gerados pelo aerolevantamento são a composição em cores verdadeiras nos canais do vermelho, verde e azul (Red, Green, Blue - RGB) e a composição colorida utilizando o infravermelho próximo (Near Infrared - NIR). O levantamento também obteve o Modelo Digital de Superfície (MDS) e o Modelo Digital de Terreno (MDT). Esses dados foram recebidos pela SDS com as etapas de pré-processamento, ajuste radiométrico, níveis de contraste, tonalidade, homogeneização das imagens, balanceamento de cores e ortorretificação já realizadas (ENGEMAP, 2012).

Os dados de entrada utilizados no presente estudo compreendem as ortoimagens correspondentes às bandas espectrais do visível e infravermelho próximo, e o MDS, formando um conjunto de dados contendo sete bandas. As ortoimagens foram recortadas em 1.500x1.500 pixels, visando maior agilidade e redução do custo computacional na aplicação das metodologias propostas. Além disso, alguns dos aplicativos utilizados, como o Segmentation Parameters Tuner (SPT) e o InterIMAGE, apresentam limitações ao trabalharem com imagens de grandes dimensões espaciais nos casos de processamento em computadores convencionais, que não dispõem de placa gráfica para processamento e/ou de grande número de processadores paralelos e quantidade excedente de memória volátil (RAM).

Ponzoni et al. (2012) falam da necessidade da conversão dos números digitais (ND) das imagens para valores de reflectância de superfície, para possibilitar a caracterização espectral dos alvos, já que um valor de ND de uma imagem em uma banda específica não está na mesma escala de outro ND de outra imagem ou outra banda espectral. Entretanto, não foi possível adquirir informações detalhadas em relação à calibração do sensor SAAPI ou do aerolevantamento (ENGEMAP, 2012).

A definição da verdade terrestre foi feita através da fotointerpretação das ortoimagens utilizando o MDS e a ferramenta 3D Analyst do ArcGIS. As classes identificadas visualmente nas imagens na área-teste A foram: vegetação em estádio inicial (VEI), médio (VEM) e avançado (VEA), plantios de Pinus spp., agricultura, campo, campo sujo (i.e., campo com vegetação arbustiva esparsa) e sombra. A área-teste $B$ possui menos classes que a área $A$, sendo somente a vegetação nos três estádios sucessionais (i.e., VEA, VEM e VEI), campo e sombra. A caracterização da vegetação também considerou dados de campo obtidos no Inventário Florístico Florestal de SC (VIBRANS et al., 2012) para a área-teste A, e dados de Faxina (2014) para a área-teste B. Nestes levantamentos, o estádio da vegetação foi definido conforme os critérios da Resolução CONAMA nº 04/94 (BRASIL, 1994).

A segmentação das imagens foi feita com o algoritmo proposto por Baatz e Schäpe (2000), escolhido por sua velocidade de execução e capacidade de extração de objetos homogêneos em uma mesma escala. Para executá-lo, são estabelecidos parâmetros de cor, forma e escala. Para a escolha destes parâmetros, foram avaliados nove cenários de segmentação, compostos pela combinação de três fatores de escala (60, 80 e 100), com pesos de cor e forma alternando entre 0,3, 0,5 e 0,7.

A avaliação dos resultados da segmentação foi feita no aplicativo SPT (ACHANCCARAY DIAZ, 2014), através de uma função de aptidão, ou métrica, que indica a qualidade da segmentação em função dos segmentos de referência criados pelo usuário. Neste trabalho, optou-se pelo Reference Bounded Segments Booster (Assistente para Segmentos Delimitados como Referência - RBSB), métrica proposta por Feitosa et al. (2006), utilizada por Novack (2009), que corresponde à razão entre a área de dois segmentos fora da interseção com a área de referência. Quanto mais próximo de zero, mais a segmentação gerada se aproxima da segmentação de referência, sendo que zero corresponde ao ajuste perfeito. A métrica RBSB mostra boa correlação com a percepção humana de qualidade de segmentação (FEITOSA et al., 2006).

Para uma das classificações, usou-se o InterIMAGE, que é um software de domínio público e código aberto (COSTA et al., 2008). Ele dispõe do algoritmo C4.5, que possibilita que a classificação baseada em objeto seja automatizada, e não somente através da rede semântica. Diferentemente do software WEKA, o InterIMAGE não oferece ao analista a possibilidade de manipular o tamanho da árvore de decisão, gerando árvores de diversas dimensões (RODRIGUES, 2014). 
O primeiro procedimento realizado no InterIMAGE foi a construção da rede semântica, que representa as classes que se espera encontrar na cena. Neste trabalho, criaram-se redes operacionais, ou seja, sem relação hierárquica entre as classes, já que o objetivo foi explorar a classificação automatizada. Desta forma, cada classe (nó-folha) foi associada ao mesmo nó-pai, sem níveis intermediários.

Na ferramenta Samples editor (editor de amostras), foi feita a segmentação das imagens com o algoritmo Baatz e Schäpe, e procedeu-se à coleta aleatória das amostras. As amostras foram coletadas de forma a abranger qualquer variação interna das classes quanto à cor, tonalidade, forma, textura e brilho, e também considerando a representatividade da classe nas imagens.

Em seguida, foi realizada a extração de atributos de cada segmento para serem usados na classificação. Foram gerados 47 atributos, sendo 43 deles espectrais e quatro operações entre bandas espectrais. Maiores detalhes sobre os atributos utilizados podem ser encontrados em Sothe (2015). Priorizaram-se atributos estatísticos ao invés dos espaciais, por se tratar predominantemente de áreas naturais, em que os objetos têm formas irregulares. Como citado por Yu et al. (2006), diferentemente da classificação de áreas urbanas, características geométricas têm pouca contribuição para a classificação da vegetação em imagens de alta resolução espacial, já que esta não possui um padrão espacial óbvio que poderia ser evidenciado na classificação.

A classificação foi feita com o algoritmo top down TA_C45_Classifier, que utiliza o conceito de árvore de decisão proposto por Quinlan (1993). O operador TA_C45_Classifier foi associado a apenas um dos nós-filho, correspondente à classe VEA da rede semântica de cada área-teste. Neste nó, habilitou-se a opção Multiclass, ficando responsável por repassar as hipóteses aos demais nós da rede. A todos os nós restantes, atribuiu-se o operador Dummy top-down. Nele, nenhuma hipótese é criada, e as informações são apenas repassadas de nó-pai para nó-filho (RODRIGUES, 2014). A classificação final resulta em um arquivo shape, e a árvore de decisão é gerada em um arquivo de texto .txt.

Adicionalmente, usou-se a ferramenta WEKA, que incorpora um conjunto de algoritmos de aprendizado de máquina que possibilita a extração do conhecimento. A metodologia desenvolvida no WEKA compreendeu as etapas de seleção de atributos e geração de modelo de classificação por árvore de decisão. O banco de dados utilizado nesta tarefa foram as amostras e respectivos atributos gerados no InterIMAGE convertido para o formato Attribute-Relation File Format (Formato de Arquivo Atributo-Relação- ARFF).

Após o procedimento de conversão, procedeu-se à seleção de atributos, envolvendo duas ferramentas a serem escolhidas pelo usuário: o avaliador de atributos e o método de busca. O avaliador determina qual método é usado para atribuir um valor a cada subconjunto de atributos, e o método de busca determina o tipo de busca a ser realizada. Utilizou-se o avaliador Correlation-based Feature Selection (Seleção de Atributos baseada em Correlação- CFS) associado ao método de busca Best-first (método da melhor busca inicial). O CFS considera um conjunto de atributos "bom", quando contém atributos altamente correlacionados com a classe e não-correlacionados entre si. A base deste método é uma heurística de avaliação de subconjuntos que considera não somente a utilidade de atributos individuais, mas também o nível de correlação entre eles (KAREGOWDA et al., 2010).

A etapa seguinte à seleção de atributos foi a classificação supervisionada do banco de dados. Optou-se pela Árvore de Regressão e Classificação (Classification and Regression Trees- CART), proposta por Breiman et al. (1984), denominada SimpleCart no WEKA. Dentre os algoritmos de árvore de decisão, este foi o que gerou árvores de melhor acurácia e menor dimensionalidade.

Para proceder com a construção do modelo WEKA no QGIS, primeiramente converteu-se a árvore de decisão em regras, separando-as conforme a classe de uso e cobertura da terra à qual pertenciam. Utilizou-se no QGIS o arquivo shape resultante da segmentação e a extração de atributos, ambos gerados no InterIMAGE, correspondente a cada área-teste. Na tabela de atributos desse arquivo, selecionaram-se as feições que satisfaziam às regras correspondentes a cada classe, e o campo "class" era preenchido conforme a classe. Esse processo repetiu-se, mudando-se as regras de decisão conforme a classe. Assim, ao final do processo, todos os segmentos estavam rotulados com as respectivas classes.

Por fim, para a avaliação dos resultados, foram geradas matrizes de confusão, a partir de amostras aleatórias geradas no QGIS sobre os mapas de referência. O número de amostras variou conforme a área ocupada pela classe em cada imagem, porém, buscou-se obedecer ao número mínimo de 50 amostras, definido por Congalton e Green (1999). A partir das matrizes, foram calculados os seguintes índices: (a) exatidão global; (b) exatidão do produtor, (c) exatidão do usuário; (d) Kappa; e (e) Kappa condicional do usuário (Kcu) (CONGALTON; GREEN, 1999). 
Foi executado o teste $z$ para testar a significância estatística da diferença entre as classificações resultantes de cada metodologia (CONGALTON; GREEN, 1999). Atribuiu-se um nível de significância de 5\% $(\mathrm{a}=0,05)$, com valor crítico de 1,96 , ou seja, assumiu-se que se o valor do teste $z$ fosse maior que o valor crítico haveria diferença significante entre os mapeamentos.

\section{RESULTADOS E DISCUSSÃO}

A partir do processamento no SPT, escolheram-se os parâmetros que obtiveram valores de RBSB mais próximos de 0 , ou seja, que mais se aproximaram aos segmentos de referência. $O$ fator de escala selecionado para ambas as áreas foi de 80 . O peso, tanto da cor quanto da forma, foi de 0,5 para área-teste $A$, e na área $B$, foi de 0,7 e 0,3 para cor e forma, respectivamente.

Na etapa de seleção de atributos, verificou-se que, dentre os 47 atributos gerados no InterIMAGE, o algoritmo CFS do WEKA selecionou subconjuntos com 15 atributos na área-teste A e 18 atributos para a área-teste B. A subtração entre a banda espectral correspondente à região do infravermelho próximo (IR1) pela banda correspondente à região do vermelho (R), além do Normalized Vegetation Difference Index (Índice de Vegetação por Diferença Normalizada- NDVI), obtido pela expressão $((I R 1-R) /(I R 1+R))$, foram atributos selecionados nas duas áreas-teste, mesmo tendo sido calculados em função dos ND. Apesar da impossibilidade de se realizar a conversão para valores de reflectância, percebe-se que os espectros de ND (Figura 1A) mostraram-se coerentes com os reportados em Vieira et al. (2003) e Piazza et al. (2016).
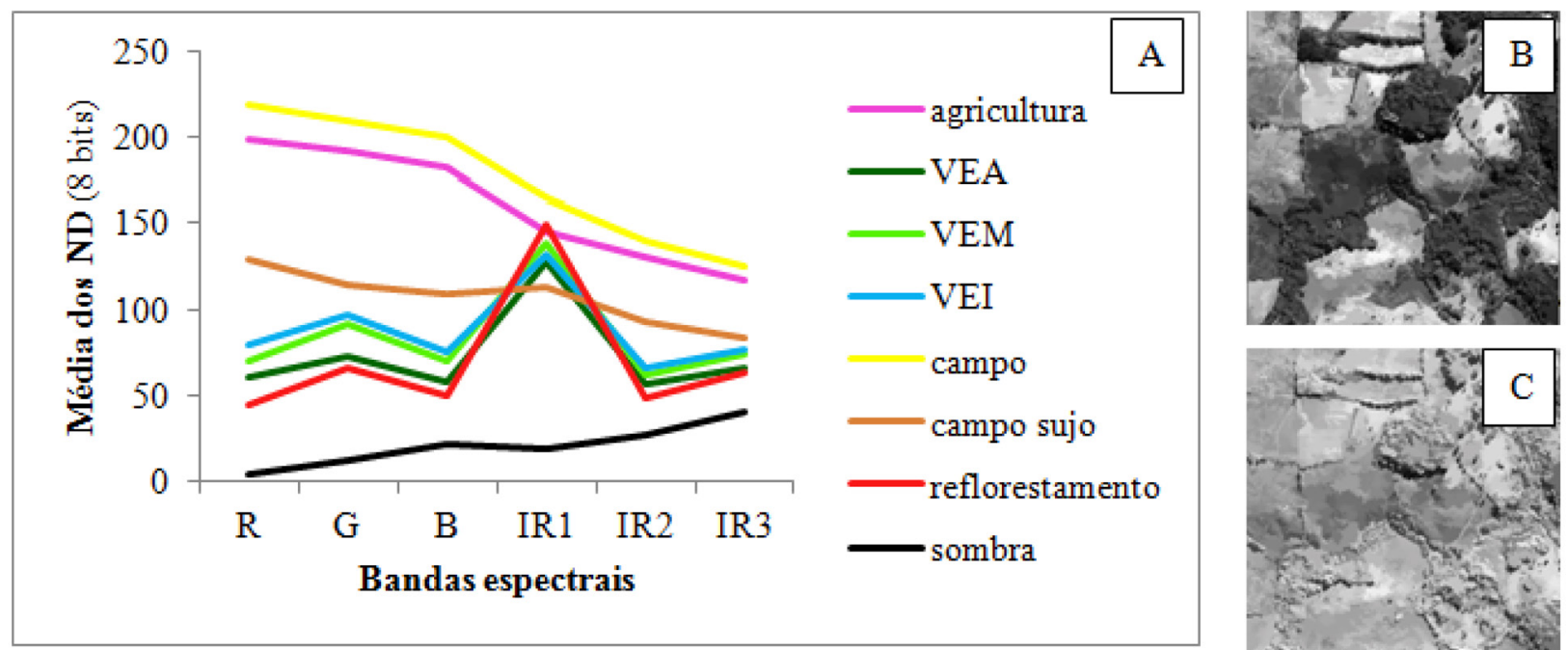

Figura 1. Perfil espectral das classes de uso e cobertura da terra da área-teste $A(A)$; espacialização da banda $R$ (B) e banda IR1 (C) na ortoimagem da área-teste A.

Figure 1. Spectral profiles for the selected land use and cover classes (A). Spatial subset of both red (B) and near-infrared spectral band images (C) for the study area A.

De acordo com Ponzoni et al. (2012), quando o dossel florestal é dividido em dois ou três estratos verticais, espera-se que ele apresente tonalidade mais escura em função das sombras dos que as demais classes da vegetação nas bandas do visível. Igualmente, espera-se ainda uma maior atividade fotossintética e tonalidade clara na banda do infravermelho próximo em razão do espalhamento múltiplo da radiação eletromagnética por parte das folhas. Entre as classes de cobertura vegetal, a VEI apresentou maiores valores de ND ao longo do espectro, em função da maior regularidade e homogeneidade dos dosséis, com exceção da região do IR1, em que VEM apresentou valores maiores que a VEI. Tal comportamento pode ser atribuído ao fato de que a VEI na área-teste A apresenta semelhanças com algumas áreas de campo sujo, classe esta que teve valores menores de ND na banda IR1. Para a VEA, observa-se que a maior heterogeneidade dos dosséis, com maior presença de sombras, resultou em ND menores que os outros estádios. A Figura 1B ilustra a espacialização das médias da banda espectral vermelho (R), em que a vegetação aparece em tons escuros, e IR1 (Figura 1C), em tons claros, indicando, portanto, valores mais altos de ND. Tal comportamento justifica o fato de a subtração entre essas duas bandas e o NDVI terem sido atributos selecionados nas duas áreas-teste. 
Outros atributos selecionados para as duas áreas-teste foram o máximo e mínimo valor de pixel do MDS correspondente a cada amostra. Como o MDS representa apenas a variação altimétrica das feições em função das altitudes, as alturas dos objetos não são conhecidas, porém, a informação de textura atrelada a essa componente é alta, desempenhando um importante papel ao discriminar as classes. Yu et al. (2006) verificaram que as características topográficas foram mais importantes para fins de discriminação da vegetação do que as características espectrais. De forma semelhante, Francisco e Almeida (2012) demonstraram a importância da inclusão de outras informações, não somente espectrais, para a discriminação das classes de cobertura da terra.

Na Tabela 1 visualizam-se as características das árvores de decisão geradas em cada um dos aplicativos. Percebe-se que, para as duas áreas, a árvore de decisão SimpleCart aliada à etapa de seleção de atributos resultou em árvores mais compactas e com menor número de atributos em comparação ao algoritmo C4.5. As operações entre as bandas do IR1 e R foram os grandes nós, ou seja, os atributos principais das árvores geradas para as duas áreas, demonstrando a relevância de tais atributos ao discriminar a vegetação das demais classes.

Tabela 1. Características das árvores de decisão geradas em cada aplicativo.

Table 1. Decision tree characteristics derived from each software.

\begin{tabular}{|c|c|c|c|c|}
\hline \multirow{2}{*}{ Software } & \multirow{2}{*}{ Algoritmo } & \multirow{2}{*}{ Característica da árvore } & \multicolumn{2}{|c|}{ Área-teste } \\
\hline & & & $\mathbf{A}$ & B \\
\hline \multirow{4}{*}{ InterIMAGE } & \multirow{4}{*}{ C4.5 } & $\mathrm{N}^{\circ}$ ramificações & 52 & 24 \\
\hline & & $\mathrm{N}^{\circ}$ atributos & 17 & 10 \\
\hline & & $N^{\circ}$ nós & 27 & 13 \\
\hline & & Atributo principal & (IR1-R) & NDVI \\
\hline \multirow{4}{*}{ WEKA } & \multirow{4}{*}{ SimpleCart } & $\mathrm{N}^{\circ}$ ramificações & 17 & 15 \\
\hline & & $\mathrm{N}^{\circ}$ atributos & 6 & 6 \\
\hline & & $\mathrm{N}^{\circ}$ nós & 10 & 8 \\
\hline & & Atributo principal & (IR1-R) & (R/IR1) \\
\hline
\end{tabular}

A Tabela 2 mostra que, para a classificação com o algoritmo C4.5 no InterIMAGE (mapa classificado A), a Exatidão Global foi de 67\% e o índice Kappa de 0,59, considerado bom de acordo com a literatura (Figura 2). Quanto aos estádios sucessionais da vegetação, os resultados podem ser considerados excelentes para as classes VEI e VEM, com Kappa condicional de 0,8, e muito bom para a VEA, com Kappa condicional de 0,6.

Na Tabela 2 também visualiza-se a matriz de confusão resultante da classificação com a árvore de decisão SimpleCart gerada no WEKA (mapa classificado B). A classificação alcançou Exatidão Global de 63\% e índice Kappa de 0,56, considerado bom. Apesar de o Kappa ser inferior ao obtido para a classificação no InterIMAGE, ambos os métodos não diferiram significantemente no teste $z$. A classe VEM apresentou o melhor resultado entre os estádios da vegetação, com Kappa condicional de 0,83. Nas duas metodologias, a classe sombra apresentou baixa exatidão e Kappa condicional do usuário, já que teve outras classes erroneamente atribuídas a ela: VEM, VEA e campo sujo, principalmente.

Outro erro de classificação recorrente na área-teste A foi observado para a classe reflorestamento (Refl.), classificada, predominantemente, como VEA. A classe VEA, nas duas metodologias, apresentou menor Kappa condicional do usuário que as demais classes de vegetação, tendo as classes VEM e reflorestamento atribuídas a ela. A VEI apresentou pouca confusão com os demais estádios de vegetação, mas foi erroneamente classificada como campo sujo pelo C4.5, e também como agricultura (Agric.) e campo pelo algoritmo SimpleCart. Tal fato deve-se à maior presença de solo na VEI. Foi verificado que a regra de decisão referente a esta classe encontra-se no mesmo ramo que as classes campo sujo e agricultura no SimpleCart, demonstrando a semelhança espectral destas classes.

O campo sujo apresenta uma variedade espectral intraclasse, já que possui áreas cobertas com uma vegetação esparsa, e outras mais "limpas", o que pode ter ocasionado a confusão desta classe com as classes campo e agricultura. Esse erro também pode estar associado ao mapa de referência da classe, já que a área classificada como campo sujo no mapa de referência, em certos locais, pode tratar-se de áreas agrícolas abandonadas. Quanto à confusão do campo sujo com a classe campo, percebe-se que ocorreram em regiões em que aquela classe possui maior brilho, semelhante ao observado por Novack (2009). O autor, ao classificar áreas urbanas com imagens WorldView, obteve confusão entre 
as classes "solo exposto" e "vegetação rasteira". Ele atribuiu isto ao fato de as duas classes estarem misturadas em alguns locais, o que descaracteriza o comportamento espectral de ambas.

Tabela 2. Matriz de confusão da classificação da área-teste A no InterIMAGE com o algoritmo C4.5 (A); e no WEKA e QGIS com o algoritmo SimpleCart (B).

Table 2. Error matrix for the classification of the study area $A$ using InterIMAGE with C4.5 algorithm (A); and for both WEKA and QGIS with the SimpleCart algorithm (B).

\begin{tabular}{|c|c|c|c|c|c|c|c|c|c|c|c|}
\hline & \multicolumn{11}{|c|}{ Mapa de referência } \\
\hline & Classe & VEI & VEM & VEA & Agric. & Campo & C. sujo & Refl. & Sombra & $\mathrm{TCl}$ & Kcu \\
\hline \multirow{11}{*}{$\begin{array}{l}\text { Mapa } \\
\text { classificado (A) }\end{array}$} & VEI & 32 & 3 & 0 & 0 & 0 & 4 & 0 & 0 & 39 & 0,80 \\
\hline & VEM & 0 & 79 & 6 & 0 & 2 & 5 & 0 & 1 & 93 & 0,81 \\
\hline & VEA & 0 & 14 & 32 & 0 & 0 & 1 & 3 & 0 & 50 & 0,60 \\
\hline & Agric. & 3 & 1 & 1 & 32 & 2 & 18 & 0 & 0 & 57 & 0,52 \\
\hline & Campo & 2 & 6 & 1 & 2 & 44 & 20 & 0 & 0 & 75 & 0,53 \\
\hline & C. sujo & 12 & 12 & 6 & 16 & 21 & 146 & 1 & 0 & 214 & 0,52 \\
\hline & Refl. & 0 & 0 & 2 & 0 & 0 & 0 & 15 & 1 & 18 & 0,83 \\
\hline & Sombra & 1 & 15 & 7 & 0 & 1 & 6 & 1 & 18 & 49 & 0,35 \\
\hline & TCo & 50 & 130 & 55 & 50 & 70 & 200 & 20 & 20 & 595 & 0,59 \\
\hline & Ep & $64 \%$ & $61 \%$ & $58 \%$ & $64 \%$ & $63 \%$ & $73 \%$ & $75 \%$ & $90 \%$ & \multirow{2}{*}{$67 \%$} & \\
\hline & $\mathrm{Eu}$ & $82 \%$ & $85 \%$ & $64 \%$ & $56 \%$ & $59 \%$ & $68 \%$ & $83 \%$ & $37 \%$ & & \\
\hline \multirow{11}{*}{$\begin{array}{l}\text { Mapa } \\
\text { classificado (B) }\end{array}$} & VEI & 30 & 5 & 2 & 0 & 2 & 13 & 1 & 0 & 53 & 0,53 \\
\hline & VEM & 1 & 85 & 5 & 0 & 2 & 4 & 0 & 1 & 98 & 0,83 \\
\hline & VEA & 1 & 12 & 34 & 0 & 0 & 5 & 8 & 1 & 61 & 0,51 \\
\hline & Agric. & 5 & 1 & 2 & 43 & 6 & 37 & 0 & 0 & 94 & 0,41 \\
\hline & Campo & 4 & 6 & 0 & 2 & 52 & 31 & 0 & 0 & 95 & 0,49 \\
\hline & C. sujo & 9 & 6 & 4 & 5 & 7 & 103 & 0 & 0 & 134 & 0,65 \\
\hline & Refl. & 0 & 0 & 1 & 0 & 0 & 0 & 10 & 0 & 11 & 0,91 \\
\hline & Sombra & 0 & 15 & 7 & 0 & 1 & 7 & 1 & 18 & 49 & 0,35 \\
\hline & TCo & 50 & 130 & 55 & 50 & 70 & 200 & 20 & 20 & 595 & 0,56 \\
\hline & Ep & $60 \%$ & $65 \%$ & $62 \%$ & $86 \%$ & $74 \%$ & $51 \%$ & $50 \%$ & $90 \%$ & \multirow{2}{*}{$63 \%$} & \\
\hline & $\mathrm{Eu}$ & $57 \%$ & $87 \%$ & $56 \%$ & $46 \%$ & $55 \%$ & $77 \%$ & $91 \%$ & $37 \%$ & & \\
\hline
\end{tabular}

Legenda: TCo: total de amostras coletadas; TCl: total de amostras classificadas; Ep: Exatidão do produtor; Ep: Exatidão do usuário; Kcu: Kappa condicional do usuário.
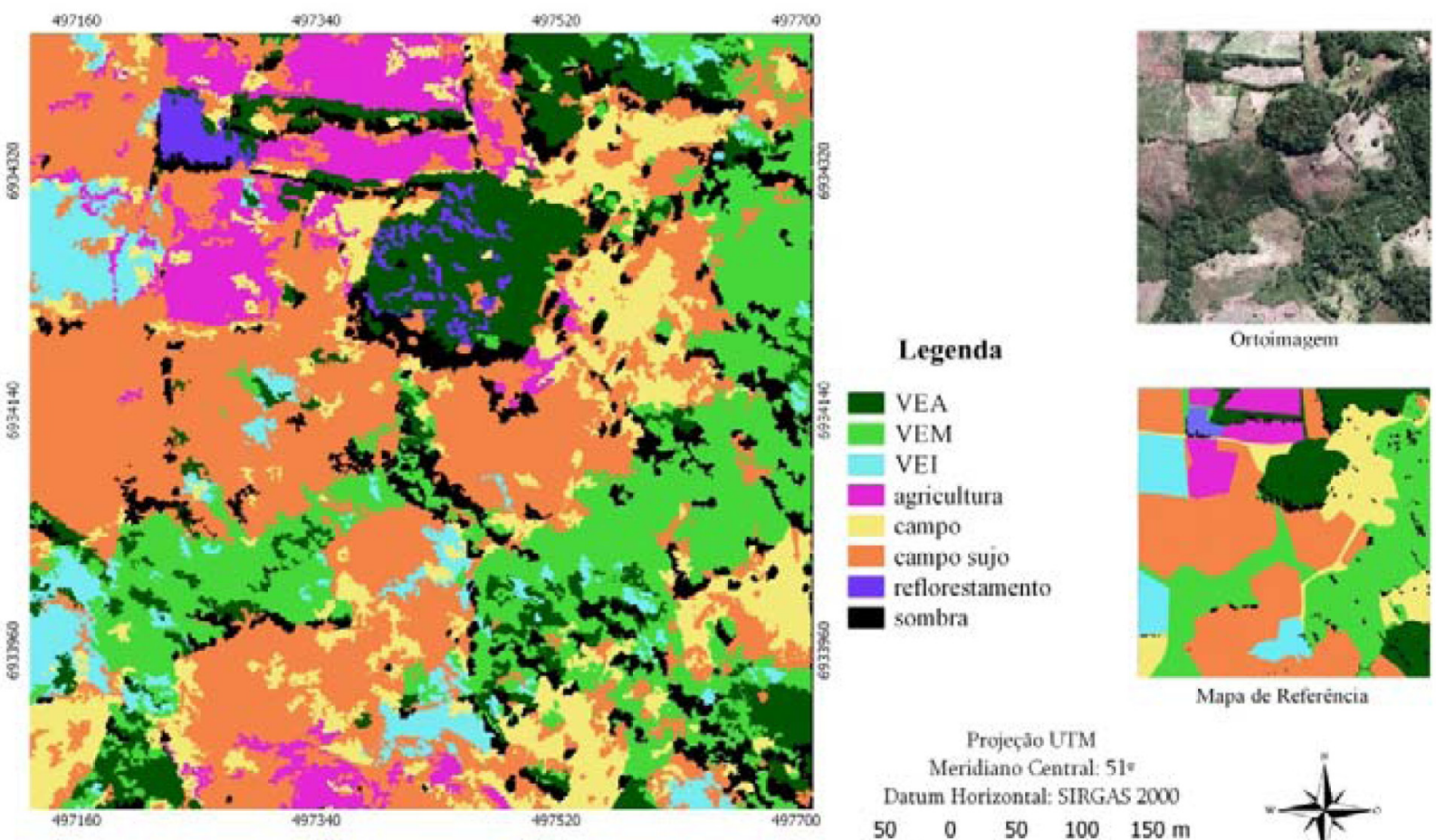

Ortuimagen

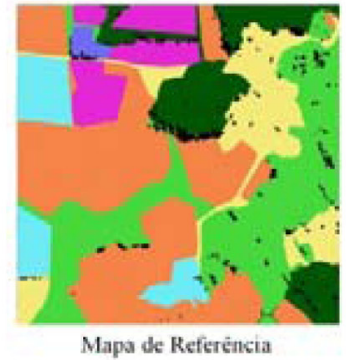

Projeção UTM

Meridiano Central: $51^{\circ}$ Datum Horizontal: SIRGAS 2000 $50 \quad 0 \quad 50 \quad 100 \quad 150 \mathrm{~m}$

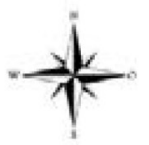

Figura 2. Mapa de uso e cobertura da terra da área-teste A gerado com o algoritmo C4.5 do InterIMAGE.

Figure 2. Land use and land cover map for the study area A generated from the InterIMAGE' C4.5 algorithm. 
Interessante que a árvore de decisão SimpleCart do WEKA com apenas uma regra de decisão para cada uma das três classes de vegetação alcançou índices de acerto considerados bons, equiparando-se ao C4.5, que utilizou de quatro a cinco regras para cada estádio. Isto demonstra que árvores de decisão generalizáveis podem trabalhar melhor com a variabilidade dos dados do que as mais complexas. Nas árvores de decisão complexas, pode ocorrer o chamado "overfitting", ou seja, um superajuste do modelo às amostras de treinamento, o que o impede de classificar corretamente as classes que não se enquadram em todos os requisitos previstos na árvore de decisão. Segundo Körting (2012), uma árvore muito grande pode superajustar os dados, enquanto uma muito pequena pode deixar de capturar estruturas importantes. Por isso, o autor diz ser preferível uma árvore média, desde que não subestime ou superestime os dados, e que também seja facilmente interpretada pelo usuário.

A Tabela 3 mostra os resultados das classificações da área-teste B com o algoritmo C4.5 no InterIMAGE (mapa classificado A) e com o SimpleCart no WEKA (mapa classificado B), que obtiveram índices Kappa de 0,53 e 0,59, respectivamente, considerados como bom. Nesta área-teste, a classificação com o algoritmo SimpleCart (Figura 3) obteve melhor desempenho que o C4.5, apesar de essas diferenças não serem significantes de acordo com o teste $z$.

Tabela 3. Matriz de confusão da classificação da área-teste $B$ no InterIMAGE com o algoritmo C4.5 (A); e no WEKA e QGIS com o algoritmo SimpleCart (B).

Table 3. Error matrix for the classification of the study area $B$ using InterIMAGE with C4.5 algorithm (A); and for both WEKA and QGIS with the SimpleCart algorithm (B).

\begin{tabular}{|c|c|c|c|c|c|c|c|c|}
\hline & \multicolumn{7}{|c|}{ Mapa de referência } & \multirow[b]{2}{*}{$\mathrm{Kcu}$} \\
\hline & Classes & VEI & VEM & VEA & Campo & Sombra & $\mathrm{TCl}$ & \\
\hline \multirow{8}{*}{ Mapa classificado $(\mathrm{A})$} & VEI & 130 & 18 & 46 & 12 & 3 & 209 & 0,41 \\
\hline & VEM & 16 & 29 & 24 & 0 & 0 & 69 & 0,36 \\
\hline & VEA & 33 & 1 & 123 & 1 & 1 & 159 & 0,62 \\
\hline & Campo & 1 & 2 & 0 & 37 & 0 & 40 & 0,92 \\
\hline & Sombra & 0 & 0 & 7 & 0 & 16 & 23 & 0,68 \\
\hline & TCo & 180 & 50 & 200 & 50 & 20 & 500 & \multirow{3}{*}{0,53} \\
\hline & Ep & $72 \%$ & $58 \%$ & $62 \%$ & $74 \%$ & $80 \%$ & $67 \%$ & \\
\hline & $\mathrm{Eu}$ & $62 \%$ & $42 \%$ & $77 \%$ & $92 \%$ & $70 \%$ & $6 / \%$ & \\
\hline \multirow{8}{*}{ Mapa classificado (B) } & VEI & 133 & 17 & 35 & 4 & 0 & 189 & 0,54 \\
\hline & VEM & 17 & 29 & 18 & 0 & 0 & 64 & 0,39 \\
\hline & VEA & 24 & 1 & 126 & 0 & 2 & 153 & 0,71 \\
\hline & Campo & 4 & 2 & 0 & 46 & 0 & 52 & 0,87 \\
\hline & Sombra & 2 & 1 & 21 & 0 & 18 & 42 & 0,40 \\
\hline & TCo & 180 & 50 & 200 & 50 & 20 & 500 & 0,59 \\
\hline & Ep & $74 \%$ & $58 \%$ & $63 \%$ & $92 \%$ & $90 \%$ & \multirow{2}{*}{$70 \%$} & \\
\hline & $\mathrm{Eu}$ & $70 \%$ & $45 \%$ & $82 \%$ & $88 \%$ & $43 \%$ & & \\
\hline
\end{tabular}

Legenda: TCo: total de amostras coletadas; TCl: total de amostras classificadas; Ep: Exatidão do produtor; Ep: Exatidão do usuário; Kcu: Kappa condicional do usuário.

Observando-se o Kappa condicional da área-teste B obtido nas duas metodologias, percebe-se que nesta área ocorreram maiores confusões entre os estádios sucessionais da vegetação, inclusive na VEI. A VEM teve várias amostras classificadas como VEI com ambos os algoritmos. Além de esta classe ser menos representativa na imagem, a VEM é um estádio de transição entre o inicial e avançado, apresentando comportamento espectral semelhante a essas duas classes. A VEA foi classificada como VEI em locais de maior brilho e, consequentemente, maiores valores da média dos ND das bandas do visível, característica dos estádios iniciais da vegetação. Por sua vez, a VEI foi classificada como VEA em locais que essa classe possui padrão espectral mais escuro.

$\mathrm{Na}$ área-teste $\mathrm{B}$ ocorreu maior confusão entre os estádios sucessionais da vegetação do que na área A. No entanto, a vegetação da área B é mais complexa, apresentando maior heterogeneidade nos diferentes estádios, além de ela estar intrinsecamente conectada e sem influências antrópicas diretas, já que se situa no interior de uma Unidade de Conservação. A VEI tem maior variabilidade espectral nesta área-teste, pois possui alguns locais onde predomina uma vegetação arbustiva, e em outros, de gramíneas. 


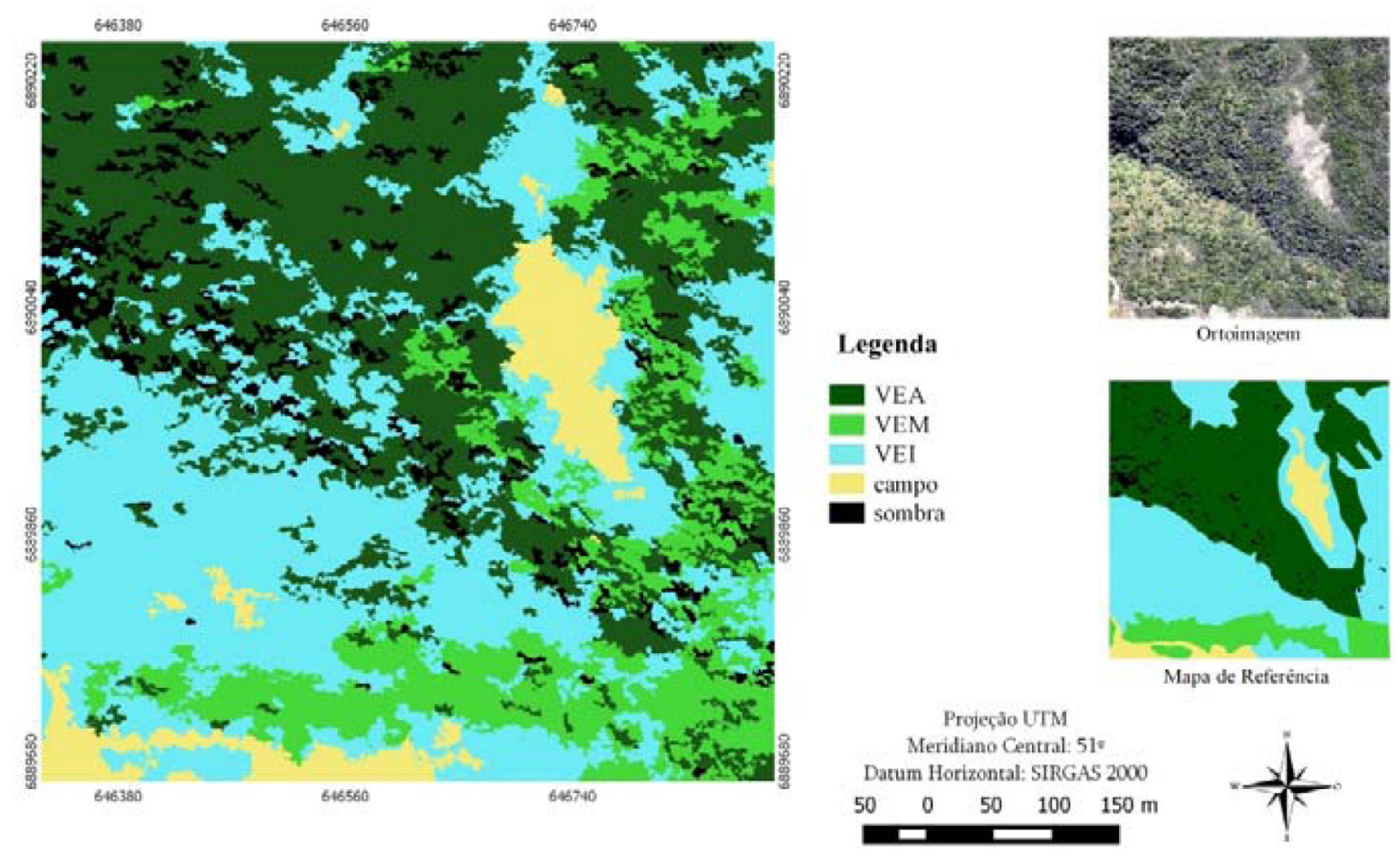

Figura 3. Mapa de uso e cobertura da terra da área-teste $B$ gerado com o algoritmo SimpleCart do WEKA. Figure 3. Land use and land cover map for the study area B generated from the WEKA' SimpleCart algorithm.

A classe sombra manteve baixos índices de exatidão do usuário e Kappa condicional do usuário nas duas áreas testadas. Porém, cabe destacar que o fotointérprete não considerou as sombras menores no interior da vegetação na elaboração do mapa de referência, apenas aquelas mais representativas. Por este motivo, essa classe teve uma superclassificação em relação ao mapa de referência, e, portanto, maiores erros de comissão.

Os resultados deste estudo ainda podem ser aprimorados aplicando-se uma heurística para reduzir erros de comissão resultantes da classificação automática, editando-se os limiares da árvore, por exemplo, e adequando-se os resultados conforme o mapa de referência. Isto constitui uma vantagem ao se utilizar algoritmos de árvore de decisão, pois, além de permitir que os limiares sejam alterados, de forma a reduzir os erros encontrados na classificação, também permite que os modelos obtidos sejam ajustados às peculiaridades da cena em análise e replicados em outras áreas com características semelhantes de uso e cobertura da terra (CAMARGO et al., 2009).

\section{CONCLUSÕES}

As técnicas de mineração de dados permitiram constatar que a vegetação apresenta características espectrais e texturais distintas conforme o seu estádio sucessional de regeneração. A etapa da seleção de atributos atendeu ao propósito de redução da dimensão e aumento da acurácia dos modelos de árvore de decisão gerados. Em geral, a componente MDS contribuiu expressivamente para a classificação de uso e cobertura da terra nas duas áreas-teste, da mesma forma que as operações entre as bandas correspondentes à região do infravermelho próximo e vermelho.

Os resultados das classificações com os algoritmos C4.5 e SimpleCart não apresentaram diferenças significantes para as duas áreas testadas. Porém, o algoritmo SimpleCart aliado à etapa de seleção de atributos, alcançou bons resultados com árvores menores e utilizando menos atributos que o C4.5.

A árvore de decisão possui a vantagem de permitir ao usuário visualizar o processo de classificação, podendo ser aperfeiçoada de forma a melhorar a acurácia de acordo com as peculiaridades de cada local. A estratégia de uso de uma estrutura hierárquica para a descrição automática das classes com base em algoritmos supervisionados de mineração de dados mostrou-se ser uma forma eficaz e de reduzida subjetividade para a classificação do uso e cobertura da terra. O fato de estas metodologias terem envolvido procedimentos automáticos faz com que sejam aplicáveis em outras áreas. 
Desta forma, a classificação automatizada mostrou-se uma alternativa interessante para auxiliar no estudo de áreas extensas e de difícil acesso, economizando assim esforço do operador bem como tempo de processamento computacional.

Este trabalho demonstrou que as metodologias testadas são aplicáveis em outras áreas do Bioma Mata Atlântica, além de ter contribuído para uma avaliação comparativa das diversas ferramentas disponíveis gratuitamente para a interpretação de ortoimagens, que podem ser acessadas no endereço sigsc.sds.sc.gov.br.

\section{REFERÊNCIAS BIBLIOGRÁFICAS}

ACHANCCARAY DIAZ, P. M. A Comparison of segmentation algorithms for Remote Sensing. 2014.84 p. Dissertação (Mestrado em Engenharia Elétrica) - Pontifícia Universidade Católica do Rio de Janeiro, Rio de Janeiro, 2014.

AMARAL, M. V. F.; SOUZA, A. L.; SOARES, V. P.; SOARES, C. P. B.; LEITE, H. G.; MARTINS, S. V.; FILHO, E. I. F.; LANA, J. M. Avaliação e comparação de métodos de classificação de imagens de satélites para o mapeamento de estádios de sucessão florestal. Revista Árvore, Viçosa, v. 33, n. 3, p. 575-82, 2009.

BAATZ, M.; SCHÄPE, A. Multiresolution segmentation - An optimization approach for high quality multiscale image segmentation. In: STROBL, J.; BLASCHKE, T.; GRIESEBNER, G. (Eds.). Angewandte Geographische Informationsverarbeitung XII. Karlsuhe: Wichmann Verlag, 2000. p. 12-23.

BLASCHKE, T.; STROBL, J. What's wrong with pixels? Some recent developments interfacing remote sensing and GIS. GIS-Zeitschrift für Geoinformationssysteme, Bonn, v. 14, n. 6, p. 12-17, 2001.

BRASIL. Conselho Nacional do Meio Ambiente. Resolução CONAMA nº 04/94, Diário Oficial da União, Brasília, n. 114. jun. 1994.

BRASIL. Lei n. 11.428 de 2006. Diário Oficial da União, Brasília, 26 dez. 2006. Seção 1, p. 1.

BREIMAN, L.; FRIEDMAN, J.; STONE, C. J.; OLSHEN, R. A. Classification and Regression Trees. Belmont: Wadsworth, 1984.

CAMARGO, F. F.; FLORENZANO, T. G.; ALMEIDA, C. M.; OLIVEIRA, C. G. Geomorphological mapping using object-based analysis and ASTER DEM in the Paraíba do Sul Valley, Brazil. International Journal of Remote Sensing, Oxon, v. 30, n. 24, p. 6613-6620, 2009.

CONGALTON, R. G.; GREEN, K. Assessing the accuracy of remotely sensed data: principles and practices. New York: Lewis Publishers, 1999.

COSTA, G. A. O. P.; PINHO, C. M. D.; FEITOSA, R. Q.; ALMEIDA, C. M.; KUX, H. J. H.; FONSECA, L. M. G.; OLIVEIRA, D. A. B. INTERIMAGE: uma plataforma cognitiva open source para a interpretação automática de imagens digitais. Revista Brasileira de Cartografia, Rio de Janeiro, v. 60, n. 4, p. 331-337, 2008.

ENGEMAP GEOINFORMAÇÃO. Relatório de produção final - edital de concorrência pública, n. 0010/2009. Florianópolis, 2012. 218 p.

FAXINA, T. C. Dilemas da regularização fundiária amigável no Parque Nacional de São Joaquim: Um estudo de caso - a valorização de áreas silvestres. 2014. 110 p. Dissertação (Mestrado em Engenharia Florestal) - Universidade do Estado de Santa Catarina, Lages, 2014.

FEITOSA, R. Q.; COSTA, G. A. O. P., CAZES, T. B.; FEIJO, B. A Genetic Approach for the automatic adaptation of segmentation parameters. In: INTERNATIONAL CONFERENCE ON OBJECT-BASED IMAGE ANALYSIS ISPRS, 2006, Salzburg. Proceedings... Salzburg: ISPRS, 2006. v. 36, n. 4/C42.

FRANCISCO, C. N.; ALMEIDA, C. M. Avaliação de desempenho de atributos estatísticos e texturais em uma classificação de cobertura da terra baseada em objeto. Boletim de Ciências Geodésicas, Curitiba, v. 18, n. 2, p. 302-326, 2012. 
HAN, J.; KAMBER, M. Data Mining: Concepts and Techniques. San Francisco: Morgan Kaufmann Publishers, 2006.

IBGE - INSTITUTO BRASILEIRO DE GEOGRAFIA E ESTATÍSTICA. Informações das cidades do Brasil, 2015. Disponível em < http://www.cidades.ibge.gov.br> Acesso em: 12 abr. 2015.

KAREGOWDA, A. G.; MANJUNATH, A. S.; JAYARAM, M. A. Comparative of attribute selection using gain ratio and correlation based feature selection. International Journal of Information Technology and Knowledge Management, Norfolk, v. 2, n. 2, p. 271-277, 2010.

KLEIN, R. M. Mapa fitogeográfico do Estado de Santa Catarina. Itajaí: Herbário Barbosa Rodrigues; Florianópolis: Universidade Federal de Santa Catarina, 1978.

KÖRTING, T. S. GeoDMA: a toolbox integrating data mining with object-based and multi-temporal analysis of satellite remotely sensed imagery. 2012. 119 p. Tese (Doutorado em Sensoriamento Remoto) - Instituto Nacional de Pesquisas Espaciais, São José dos Campos, 2012.

NOVACK, T. Classificação da cobertura da terra e do uso do solo urbano utilizando o sistema InterIMAGE e imagens do sensor. 2009. 214 p. Dissertação (Mestrado em Sensoriamento Remoto) - Instituto Nacional de Pesquisas Espaciais, São José dos Campos, 2009.

PIAZZA, G. A.; VIBRANS, A. C.; LIESENBERG, V.; REFOSCO, J. C. Object-oriented and pixel-based classification approaches to classify tropical successional stages using airborne high-spatial resolution images. GIScience \& Remote Sensing, London, v. 53, n. 2, p. 206-226, 2016.

PONZONI, F. J.; SHIMABUKURO, Y. E.; KUPLICH, T. M. Sensoriamento Remoto da Vegetação. São Paulo: Oficina de Textos, 2012.

QUINLAN, R. C4.5: programs for machine learning. San Francisco: Morgan Kaufmann, 1993.

RIBEIRO, M. C.; METZGER, J. P.; MARTENSEN, A. C.; PONZONI, F. J.; HIROTA, M. M. The Brazilian Atlantic Forest: How much is left, and how is the remaining forest distributed? Implications for conservation. Biological Conservation, Oxford, v. 142, n. 6 p. 1141-1153, 2009.

RODRIGUES, T. C. S. Classificação da cobertura e do uso da terra com imagens WorldView-2 de setores norte da Ilha do Maranhão por meio do aplicativo InterIMAGE e de mineração de dados. 2014. 87 p. Dissertação (Mestrado em Sensoriamento Remoto) - Instituto Nacional de Pesquisas Espaciais, São José dos Campos, 2014.

SOTHE, C. Classificação do estádio sucessional da vegetação empregando análise baseada em objeto e ortoimagens. 2015. 249 p. Dissertação (Mestrado em Engenharia Florestal) - Universidade do Estado de Santa Catarina, Lages, 2015.

VIBRANS, A. C.; SEVEGNANI, L.; GASPER, A. L.; LINGNER, D. V. Inventário Florístico Florestal de Santa Catarina: Diversidade e conservação dos remanescentes florestais. Blumenau: Edifurb, 2012.

VIEIRA, I. C. G.; ALMEIDA, A. S.; DAVIDSON, E. A.; STONE, T. A.; CARVALHO, C. J. R.; GUERRERO, J. B. Classifying successional forests using Landsat spectral properties and ecological characteristics in eastern Amazônia. Remote Sensing of Environment, New York, v. 87, n. 4, p. 470-481, 2003.

YU, Q.; GONG, P.; CLINTON, N.; BIGING, G.; KELLY, M.; SCHIROKAUER, D. Object-based Detailed Vegetation Classification with Airborne High Spatial Resolution Remote Sensing Imagery. Photogrammetric Engineering \& Remote Sensing, Bethesda, v. 72, n. 7, p. 799-811, 2006.

Recebido em 08/10/2015

Aceito para publicação em 26/04/2016

Sci. For., Piracicaba, v. 44, n. 112, p. 863-873, dez. 2016 DOI: dx.doi.org/10.18671/scifor.v44n112.08 
